\title{
LONGEVIDADE PÓS-COLHEITA DE INFLORESCÊNCIAS DE GIRASSOL AFETADA POR NITRATO DE PRATA E SACAROSE
}

ARTHUR DOS REIS GONZAGA ${ }^{1}$; LORENA DE ARAÚJO MOREIRA ${ }^{1}$; FLAVIA LONARDONI ${ }^{1}$ e RICARDO TADEU FARIA $^{1}$

\section{RESUMO}

O objetivo deste trabalho foi avaliar a longevidade pós-colheita de inflorescências de girassol pelo uso de sacarose (4 e 10\%) ou associadas ao nitrato de prata $\left(10\right.$ e $\left.20 \mathrm{mgL}^{-1}\right)$, e testemunha (água deionizada). Os tratamentos, com três repetições cada um, foram distribuídos em delineamento inteiramente casualizado com três inflorescências por repetição. A longevidade das inflorescências foi afetada por sacarose a $4 \%$, que aumentou até em cinco dias a vida de vaso das inflorescências em relação à testemunha. $\mathrm{O}$ nitrato de prata associado à sacarose não influenciou a longevidade.

Palavras-chave: flor de corte, conservação, qualidade.

\section{ABSTRACT \\ Sunflower postharvest longevity affected by silver nitrate and sucrose treatments}

The postharvest of sunflower inflorescences was evaluate by six treatments with sucrose (4 and $10 \%)$ or sucrose plus silver nitrate (10 and $20 \mathrm{mgL}^{-}$ $\left.{ }^{1}\right)$ and the control (deionized water). The treatments, with three replications each, were distributed in a complete randomized block design with three inflorescences per replication. Longer longevity was reached by $4 \%$ sucrose that increased the vase life of the inflorescence by up to five days compared to the control. Silver nitrate associated with sucrose did not improve the longevity.

Key words: cut flowers, conservation, quality.

\section{INTRODUÇÃO}

O girassol (Helianthus annuus), família Asteraceae, é nativo da América do Norte. Seu uso como flor de corte vem aumentando nos últimos anos, como alternativa às suas várias possibilidades econômicas. As variedades utilizadas como flor de corte surgiram a partir de cruzamentos e seleção realizados pelos geneticistas e, em alguns países, já estão disponíveis cultivares com "flores" dobradas, livres de pólen e cores variadas (RICE, 1996).

O sucesso comercial de uma nova flor de corte não depende apenas de sua qualidade estética e produção, mas também de sua vida de vaso. A longevidade pós-colheita de flores de corte pode ser melhorada pelo tratamento com conservantes que mantêm a qualidade e prolongam a vida de vaso das hastes, pelo fornecimento de açúcares e conservação da hidratação dos tecidos (HALEVY \& MAYAK, 1979).

O balanço hídrico é considerado fator determinante na longevidade dos órgãos das plantas, e a deficiência de água no organismo acelera a senescência. Altos níveis de hidratação dos tecidos são em geral associados ao aumento da vida em vaso das flores de corte, enquanto perdas de 10 a $15 \%$ de sua massa fresca podem levar à morte dos tecidos (MORAES et al., 1999). A turgescência é necessária para o desenvolvimento dos botões florais e também para continuidade da atividade metabólica da flor cortada.

Os açúcares desempenham papel importante na manutenção da qualidade de flores de corte, pois a

\footnotetext{
${ }^{1}$ Universidade Estadual de Londrina.Departamento de Agronomia, Caixa Postal 6001, 86051-990 Londrina (PR). E-mail: faria@uel.br
} 
quantidade de açúcar nelas contida é limitada (ICHIMURA, 1998). A sacarose favorece o balanço hídrico das flores cortadas (CASTRO et al., 1982), acumulando-se nas flores, aumentando a concentração de solutos osmoticamente ativos e, conseqüentemente, favorecendo a manutenção da turgescência das pétalas (HALEVY, 1976).

A sacarose pode ser aplicada como um prétratamento com solução de "pulsing", procedimento que satura os tecidos com açúcares e outros compostos químicos, prolongando a vida das flores (HALEVY \& MAYAK, 1979), ou com suplementação contínua na solução de vaso. A maioria das flores responde favoravelmente a um fornecimento contínuo na solução de vaso, mas o efeito da solução de "pulsing" varia consideravelmente, dependendo da espécie e da concentração (HAN, 1992).

O talo floral, ao ser cortado, incrementa a produção de etileno e, conseqüentemente, os órgãos reprodutivos amadurecem com maior rapidez. Essa é uma característica indesejável em flores de corte, uma vez que lhes diminui a longevidade e o valor comercial. O tratamento das flores com inibidores da síntese ou ação do etileno faz-se necessário, em plantas sensíveis ao gás, pois previne a senescência típica das pétalas. Certos sais, entre os quais se inclui o nitrato de prata, impedem a ação prejudicial do etileno tanto endógeno como exógeno (BEYER, 1977). O nitrato de prata também possui propriedades inibidoras do desenvolvimento de microrganismos que causam a oclusão dos vasos condutores, limitando a absorção de soluções.

AMAURITEI \& RADELUSCU (1982) observaram aumento na durabilidade de cravos quando mantidos em soluções contendo sacarose a $4 \%$, associada a sulfatos de alumínio e potássio como germicidas. OHKAWA et al. (1999), analisando o efeito dos compostos de prata STS e $\mathrm{AgNO}_{3}$ sobre a conservação pós-colheita de rosas cv. 'Asami Red', observaram aumento da vida de vaso e redução da incidência de bent neck (anelamento e tombamento).

Não se encontraram na literatura, informações relativas à pós-colheita de inflorescências de girassol utilizando-se sacarose e nitrato de prata. $\mathrm{O}$ objetivo deste trabalho foi avaliar o efeito dessas substâncias, em diferentes concentrações, sobre a longevidade de vaso das inflorescências de girassol.

\section{MATERIAL E MÉTODOS}

Colheram-se as inflorescências em Holambra (SP) em abril de 2000, no ponto de colheita do capítulo totalmente aberto, floração plena, estádio R5,5 (quando $50 \%$ das flores do capítulo estão abertas, lígulas expandidas) conforme CASTIGLIONI et al. (1997). Foram embarcadas para Londrina (PR) em caminhão climatizado a $5-7^{\circ} \mathrm{C}$, poucas horas após colhidas, armazenadas em câmara fria a $7^{\circ} \mathrm{C}$ por quatro dias e retiradas momentos antes do início do experimento. Depois da abertura dos pacotes, fez-se a retirada das folhas, pois o girassol é comercializado na maioria das floriculturas brasileiras sem as mesmas. Em seguida, efetuou-se o corte das hastes em bisel sob água corrente, deixando-as com $50 \mathrm{~cm}$ de comprimento. Os tratamentos utilizados constam da Tabela 1. Em todos os tratamentos foram acrescentadas duas a três gotas de hipoclorito de sódio $(2,0-2,5 \%)$ às soluções.

Tabela 1.Tratamentos com diferentes concentrações de sacarose e nitrato de prata utilizados para conservação das inflorescências de girassol

\begin{tabular}{|c|c|c|}
\hline Tratamentos & $\begin{array}{c}\text { Sacarose } \\
(\mathrm{p} / \mathrm{v})\end{array}$ & $\mathrm{AgNO}_{3}$ \\
\hline & \multicolumn{2}{|c|}{ Concentrações } \\
\hline $\mathrm{T} 1 \ldots \ldots \ldots \ldots \ldots$ & - & - \\
\hline $\mathrm{T} 2 \ldots \ldots \ldots \ldots \ldots$ & $4 \%$ & - \\
\hline $\mathrm{T} 3 \ldots \ldots \ldots \ldots \ldots \ldots$ & $4 \%$ & $10 \mathrm{mgL}^{-1}$ \\
\hline $\mathrm{T} 4$..................... & $4 \%$ & $20 \mathrm{mgL}^{-1}$ \\
\hline T5 ................... & $10 \%$ & - \\
\hline T6 ...................... & $10 \%$ & $10 \mathrm{mgL}^{-1}$ \\
\hline $\mathrm{T} 7$..................... & $10 \%$ & $20 \mathrm{mgL}^{-1}$ \\
\hline
\end{tabular}

O experimento foi realizado em sala do Laboratório de Fitotecnia do Departamento de Agronomia da Universidade Estadual de Londrina (PR). Transcorreram quatro dias entre colheita, armazenamento e inicio do experimento. As inflorescências foram distribuídas em delineamento inteiramente casualizado, com sete tratamentos e três repetições, contendo três inflorescências em cada recipiente com capacidade para dois litros. A cada dois dias, as soluções foram renovadas. A temperatura da sala oscilou entre $22-24^{\circ} \mathrm{C}$, sendo a umidade relativa de $60 \%$. As avaliações foram diárias, por um 
período de nove dias, sob critério de notas (CASTRO et al., 1984), definido para comparar e verificar a conservação dos aspectos visuais necessários à comercialização, conforme Figura 1. As notas obtidas foram submetidas à análise da variância e as médias, comparadas pelo teste de Tukey (5\%).

\section{RESULTADOS E DISCUSSÃO}

$\mathrm{Na}$ Tabela 2, utilizando o critério de notas, encontram-se os resultados obtidos no experimento com os diferentes tratamentos para qualidade póscolheita de inflorescências de girassol.

Observou-se que, até o terceiro dia, não houve diferença significativa entre os tratamentos. No $5 .^{\circ}$ dia, o tratamento T2 (sacarose 4\%) apresentava nota três, enquanto o T1 (testemunha), T4 (sacarose 4\% + $20 \mathrm{mgL}^{-1} \mathrm{AgNO}_{3}$ ), $\mathrm{T} 6$ (sacarose 10\% + $10 \mathrm{mgL}^{-1}$ $\mathrm{AgNO}_{3}$ ) e $\mathrm{T} 7$ (sacarose $10 \%+20 \mathrm{mgL}^{-1} \mathrm{AgNO}_{3}$ ) apresentavam notas inferiores a dois, com evidentes sinais de senescência e perda de qualidade das inflorescências. No $8^{\circ}$ dia, as inflorescências no T2 apresentavam nota acima de 2,5, demonstrando a eficiência do tratamento com sacarose a $4 \%$.

MORAES et al. (1997), testando o efeito do "pulsing" com sacarose sobre o índice de sobrevivência de margaridas, da mesma família do girassol, obtiveram bons resultados com solução de sacarose a $10 \%$, por doze horas, alcançando índice de sobrevivência de 35,8 neste tratamento, contra 14,9 da testemunha.

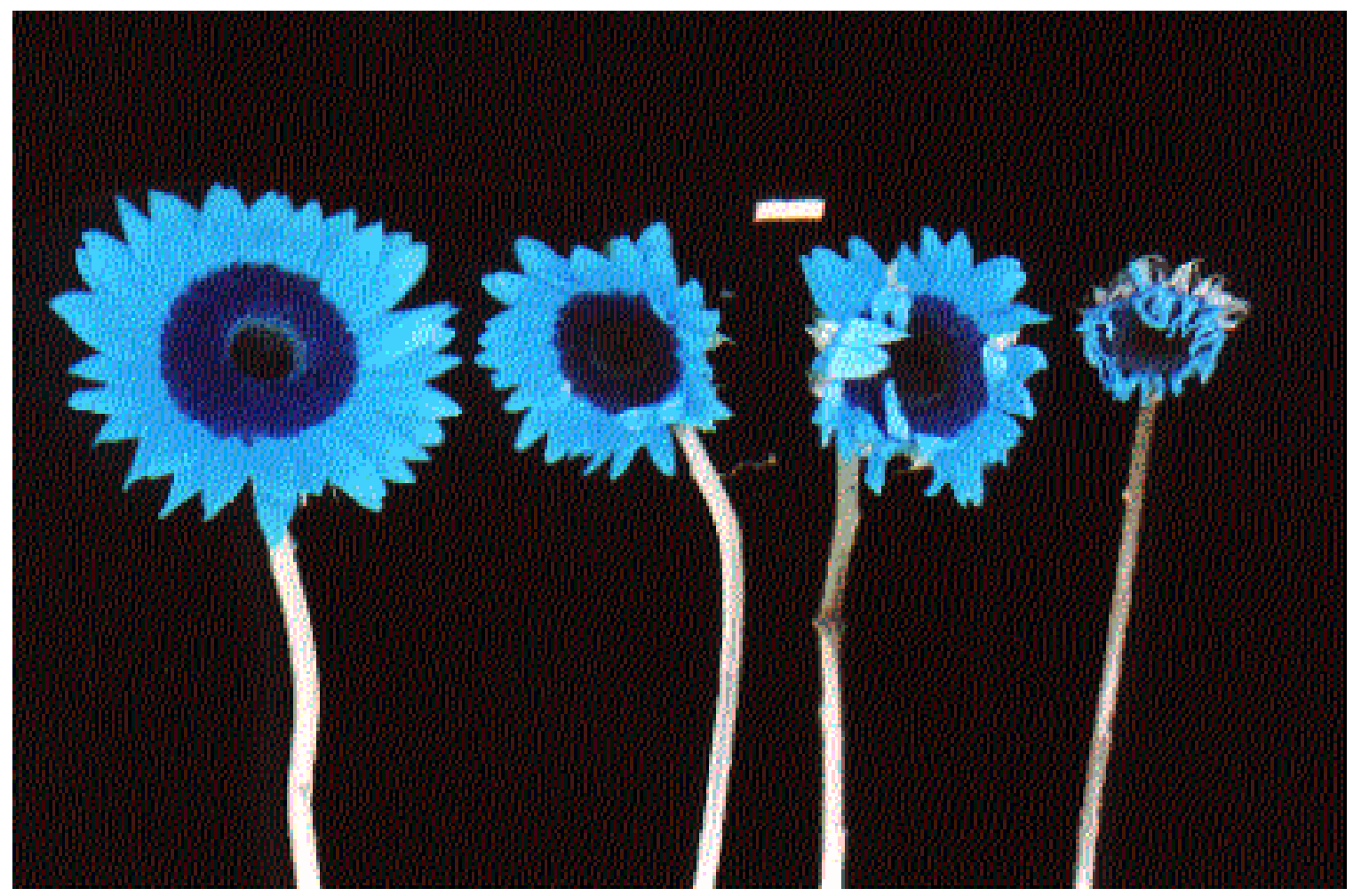

Figura 1. Critério de classificação por notas para avaliação da qualidade e longevidade das inflorescências de girassol. Nota 3 = inflorescência com características perfeitas para comercialização, túrgida, vistosa e sem manchas; nota 2 = inflorescência com alguma característica alterada, mas ainda com qualidade comercial; nota 1 = inflorescência com lígulas murchas e/ou manchadas, não adequada para comercialização, e nota 0 = inflorescência com lígulas ressecadas ou sem as mesmas. 
Tabela 2. Notas obtidas na avaliação dos tratamentos com sacarose e nitrato de prata na conservação póscolheita de inflorescências de girassol. O 1. ${ }^{\circ}$ dia corresponde a 4 dias após a colheita. As flores foram armazenadas a $5-7^{\circ} \mathrm{C}$, seguido de temperaturas de $22-24^{\circ} \mathrm{C}$

\begin{tabular}{|c|c|c|c|c|c|c|c|}
\hline \multirow{2}{*}{ Tratamentos } & \multicolumn{7}{|c|}{ Dias } \\
\hline & $1 . \circ$ & $2 .^{\circ}$ & $3 .{ }^{\circ}$ & $5 .^{\circ}$ & $7 .^{\circ}$ & $8 .^{\circ}$ & $9 .^{\circ}$ dia \\
\hline $\begin{array}{c}\left(\mathrm{Sac} / \mathrm{AgNO}_{3}\right) \\
\left(\% / \mathrm{mgL}^{-1}\right)^{3}\end{array}$ & & & & Notas & & & \\
\hline $\mathrm{T} 1(0 / 0) \ldots \ldots \ldots . .$. & $3,00 \mathrm{a}^{1}$ & $3,00 \mathrm{a}$ & $2,67 \mathrm{a}$ & $1,22 \mathrm{~d}$ & $0,33 c$ & $0,00 \mathrm{c}$ & $0,00 \mathrm{~b}$ \\
\hline $\mathrm{T} 2(4 / 0) \ldots \ldots \ldots . .$. & $3,00 \mathrm{a}$ & $3,00 \mathrm{a}$ & $3,00 \mathrm{a}$ & $3,00 \mathrm{a}$ & $2,78 \mathrm{a}$ & $2,55 \mathrm{a}$ & $1,77 \mathrm{a}$ \\
\hline $\mathrm{T} 3(4 / 10) \ldots \ldots \ldots$ & $3,00 \mathrm{a}$ & $3,00 \mathrm{a}$ & $2,78 \mathrm{a}$ & $2,67 \mathrm{ab}$ & $1,55 b$ & $1,22 b$ & $0,44 b$ \\
\hline $\mathrm{T} 4(4 / 20) \ldots \ldots \ldots$ & $3,00 \mathrm{a}$ & $3,00 \mathrm{a}$ & $2,67 \mathrm{a}$ & $1,55 \mathrm{~cd}$ & $1,11 b$ & $0,89 \mathrm{~b}$ & $0,66 b$ \\
\hline T5 $(10 / 0) \ldots \ldots . .$. & $3,00 \mathrm{a}$ & $3,00 \mathrm{a}$ & $2,78 \mathrm{a}$ & $2,33 \mathrm{abc}$ & $1,44 b$ & $0,89 \mathrm{~b}$ & $0,44 b$ \\
\hline T6 $(10 / 10) \ldots \ldots$. & $3,00 \mathrm{a}$ & $3,00 \mathrm{a}$ & $2,78 \mathrm{a}$ & $1,88 \mathrm{bcd}$ & $1,11 \mathrm{~b}$ & $0,66 b c$ & $0,44 b$ \\
\hline T7 $(10 / 20) \ldots \ldots$ & $3,00 \mathrm{a}$ & $3,00 \mathrm{a}$ & $2,67 \mathrm{a}$ & $1,99 \mathrm{~cd}$ & $1,22 b$ & $0,55 \mathrm{bc}$ & $0,33 b$ \\
\hline Médias .............. & 3,00 & 3,00 & 2,76 & 2,09 & 1,36 & 0,96 & 0,58 \\
\hline DMS ................... & 0,00 & 0,00 & 3,47 & 1,05 & 0,70 & 0,75 & 0,73 \\
\hline
\end{tabular}

${ }^{1}$ Médias seguidas pela mesma letra na vertical, não diferem entre si pelo teste de Tukey a 5\% de significância.

Por seu lado, AMAURITEI \& RADELUSCU (1982) observaram aumento na durabilidade de cravos cv. 'White Sim', pelo fornecimento contínuo de soluções contendo sacarose a 4\%. Para o girassol, o fornecimento contínuo de solução de sacarose a $4 \%$, aumentou a longevidade e qualidade das inflorescências significativamente. LONARDONI \& FARIA $^{2}$, avaliando o efeito do pulsing na qualidade pós-colheita de inflorescências de girassol, obtiveram bons resultados com soluções de sacarose a $5 \%$ por vinte e quatro horas. Segundo CASTRO (1984), a manutenção da qualidade floral ocorre em função do fornecimento de açúcares exógenos que mantêm o volume da matéria seca das flores cortadas e o nível de substratos respiratórios, especialmente nas pétalas, conservando a respiração vital e prolongando a longevidade. BHATTACHARJEE (1998) em experimento de conservação de Rosa híbrida cv. 'Happiness', testando diversas fontes de açúcar (sacarose, D-frutose, D-manose, dextrose, maltose e lactose), constatou que, com exceção do tratamento com D-manose, todas as outras fontes de açúcar foram eficientes no aumento da vida pós colheita, peso da massa fresca e seca das flores.
A utilização da sacarose associada ao nitrato de prata não apresentou resultados favoráveis quanto à manutenção da qualidade comercial das inflorescências de girassol em nenhum dos tratamentos (Tabela 2). Aqueles com sacarose isoladamente, mostraram-se superiores quando comparados aos tratamentos associados ao nitrato de prata. CASTRO et al. (1982) observaram em rosas cv. 'Pascali' que houve redução do volume absorvido da solução de vaso quando eram aumentadas as concentrações de nitrato de prata. Os autores atribuíram o fato a um possível bloqueio dos vasos condutores das hastes florais pelo nitrato de prata. A utilização do íon prata sob outra forma seria uma alternativa, uma vez que, na forma de nitrato, esse íon é relativamente imóvel nas hastes e seu local de ação se situa provavelmente, no corte ou próximo a este (PAUL \& GOO, 1982).

\section{CONCLUSÕES}

1. O tratamento com sacarose a $4 \%$ proporcionou maior longevidade às inflorescências

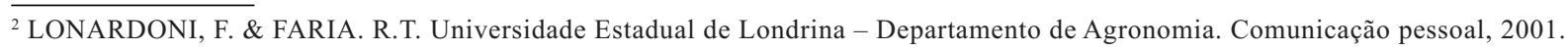


de girassol, aumentando até em cinco dias a vida de vaso em relação à testemunha.

2. A utilização da sacarose associada ao nitrato de prata não foi eficiente em estender a vida de vaso das flores, nas concentrações testadas.

\section{LITERATURA CITADA}

AMAURITEI, A. \& RADELUSCU, I. Rezultate cu privire la pastrarea garoafelor in solutti conservante la temperatura mediuli ambiant. Lucr. Stiint., v.13, p.8389, 1982.

BEYER. E. M. ${ }^{14} \mathrm{C}_{2} \mathrm{H}_{4}$ : Its incorporation and oxidation on to ${ }^{14} \mathrm{CO}_{2}$ by cut Carnation. Plant Physiology, v. 60, p.203206, 1977.

BHATTACHARJEE, S. K. Evaluation of different types of sugar for improving post harvest life and quality of cut roses. Annals of Agricultural Research, v. 2 0, .n. 2, p. 159-165, 1998.

CASTIGLIONI, V. B. R. et al. Fases do desenvolvimento da planta de girassol. Londrina: EMBRAPA-CNPSo, 1997. 24p. (EMBRAPA-CNPSo. Documentos, 58).

CASTRO, C. E. F. de Tratamentos químicos pós-colheita e critérios de avaliação de qualidade de cravos Dianthus caryophyllus cv. Scania Red Sim. Piracicaba, 1984. 139p. Dissertação (Mestrado em Fitotecnia) Escola Superior de Agricultura "Luiz de Queiroz".

CASTRO, C. E. F. de; VIDIGAL, J. C. \& GARCIA, J. L. M. Efeito da sacarose e do nitrato de prata na durabilidade de rosas cultivar 'Pascali'. Revista Horticultura, p.161177, 1982.
HALEVY, A. H. Treatments to improve water balance of cut flowers. Acta Horticulturae, Aas. Sweden, v.64, p. 223230, 1976.

HALEVY, A.H. \& MAYAK, S. Senescence and post harvest physiology of cut flowers - part 1. Hortic. Rev., Westport., v.1, p.204-236, 1979.

HAN, S. S. Role of sucrose in bud development and vase life of cut Liatris spicata (L.) Willd. HortSciense, Massachusetts, v. 27, n.11, p.1198-1200, 1992.

ICHIMURA, K. Improvement of post harvest life in several cut flowers by the addition of sucrose. JARQ - JapanAgricultural-Research-Quarterly, v. 32, n.4, p.275280, 1998.

MORAES, P. J. et al. Efeito da refrigeração e do "pulsing" com sacarose sobre o teor relativo de água nas sépalas das flores de ave-do-paraiso (Strelitzia reginae Ait.) In: CONGRESSO BRASILEIRO DE FLORICULTURA E PLANTAS ORNAMENTAIS, 12., Jaboticabal, 1999. Resumos... Jaboticabal, 1999. p.33.

MORAES, P. J. et al. Efeito do "pulsing" com sacarose sobre o Índice de Sobrevivência de Chrysanthemum leucanthemum L. Rev. Bras. Hortic. Ornam., Campinas, v.3, n.2, p.80-84, 1997.

OHKAWA, K. et al. Mobility and effects on vase life of silvercontaining compounds in cut rose flowers. HortScience, v. 34, n. 1, p.112-113, 1999.

PAUL, R.E. \& GOO, T.T.C. Pulse treatment with silver nitrate extends vase life of Anthuriums. J. Amer. Soc. Hort. Sci, Honolulu, v.107, n.5, p. 842-844, 1982.

RICE, G. Rays of sunshine. Garden London, London, v.121, n.8, p.490-495, 1996. 